\title{
On the galloping instability of two-dimensional bodies having elliptical cross-sections
}

\author{
G. Alonso , J. Meseguer, A. Sanz-Andrés, E. Valero \\ IDR/UPM, E.T.S.J. Aeronáuticos, Universidad Politécnica de Madrid, E-28040 Madrid, Spain
}

\begin{abstract}
A B S T R A C T
Galloping, also known as Den Hartog instability, is the large amplitude, low frequency oscillation of a structure in the direction transverse to the mean wind direction. It normally appears in the case of bodies with small stiffness and structural damping, when they are placed in a flow provided the incident velocity is high enough. Galloping depends on the slope of the lift coefficient versus angle of attack curve, which must be negative. Generally speaking this implies that the body is stalled after boundary layer separation, which, as it is known in non-wedged bodies, is a Reynolds number dependent phenomenon. Wind tunnel experiments have been conducted aiming at establishing the characteristics of the galloping motion of elliptical cross-section bodies when subjected to a uniform flow, the angles of attack ranging from $0^{\circ}$ to $90^{\circ}$. The results have been summarized in stability maps, both in the angle of attack versus relative thickness and in the angle of attack versus Reynolds number planes, where galloping instability regions are identified.
\end{abstract}

Galloping

Elliptical cross-section bodies

Wind tumnel density; $U$ the upstream flow velocity, and $b$ is a cross-flow characteristic length of the body under consideration.

Eq. (1) is obtained after linearization of the motion equation considering the action of the aerodynamic forces in the direction normal to the incident wind, and is closely related to the sign of the lift coefficient slope at the reference angle. Negative values of such slope may lead to potentially unstable situations. Galloping instability can be explained in terms of the flow pattern around the body. In the non-separated flow condition, the lift coefficient increases with the angle of attack, hence the lift slope becomes generally positive and galloping does not happen. However, when the angle of attack increases beyond a threshold value, boundary layer separates and then the lift coeficient decreases. Therefore, after stalling the lift coeficient slope becomes negative and according to Eq. (1) the possibility of galloping oscillations appears. Obviously, the stalling can be very different depending on the character (laminar or turbulent) of the flow in the boundary layer before separation. Since the main parameters affecting such a boundary layer characteristic are the upstream Reynolds number and the upstream turbulence, they have utmost relevant influence in the stalling behaviour and, therefore, they can play a significant role in galloping phenomena.

Galloping is a low frequency phenomenon, that takes place at much lower frequencies than vortex shedding. In addition galloping instability is caused by the change with the body angle of attack of aerodynamic forces, whereas vortex shedding is an inherent characteristic of the body wake formation. Therefore, although in very specific circumstances both phenomena can 
appear simultaneously, they generally are uncoupled and can be analysed separately (Blevins, 2001; Naudascher and Rockwell, 1994).

The prediction of the galloping amplitude has relied, so far, on the determination of the static aerodynamic coefficients, which can be obtained using wind tunnel experiments. To asses the suitability of the static tests approach to support galloping analyses, both static and dynamic wind tunnel tests were performed (Alonso et al., 2005, 2007) showing that the results obtained from the static tests are in very good agreement with the dynamic ones.

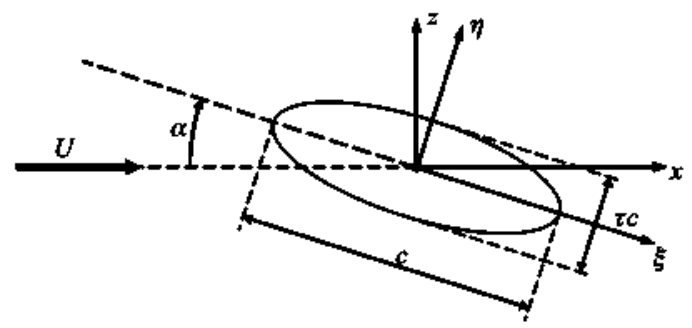

Fig. 1. Definition of the main parameters involved in the galloping of elliptical cross-section cylinders.
In the last decades large efforts have been devoted to experimentally study the galloping features of many bodies having different cross-sections. A review of the literature dealing with this topic, including the stabilizing effects of turbulence in some cases, can be found in Hêmon and Santi (2002). However, most of those efforts have been focused on bodies with square or rectangular cross-sections, although prismatic bodies with other also interesting cross-sectional shapes can be unstable to transverse galloping (Blevins, 2001). A general analysis based on Glauert-Den Hartog criterion of two-dimensional triangular crosssectional bodies (the main vertex angle $\beta$ ranging from $10^{\circ}$ to $90^{\circ}$ ) can be found in Alonso and Meseguer (2006), and a similar study but based on dynamical tests is reported in Alonso et al. (2005, 2007), although these last studies are limited to the range $10^{\circ} \leq \beta \leq 60^{\circ}$.

Galloping usually occurs in pylons of suspension and cable stayed bridges, ice-coated power lines and cables, and in structures with non-symmetrical cross-sections (obviously circular cylinders are not susceptible to galloping-induced vibrations because they are symmetric, and then $d c_{i} / d \alpha_{c}=0$, independently of the value of the angle of attack). Because of that, most of the studies related to galloping are concerned with edged crosssection bodies, where the position of the boundary layer detachment point can be clearly anticipated. This is no longer valid in the case of bodies having a rounded, smooth surface
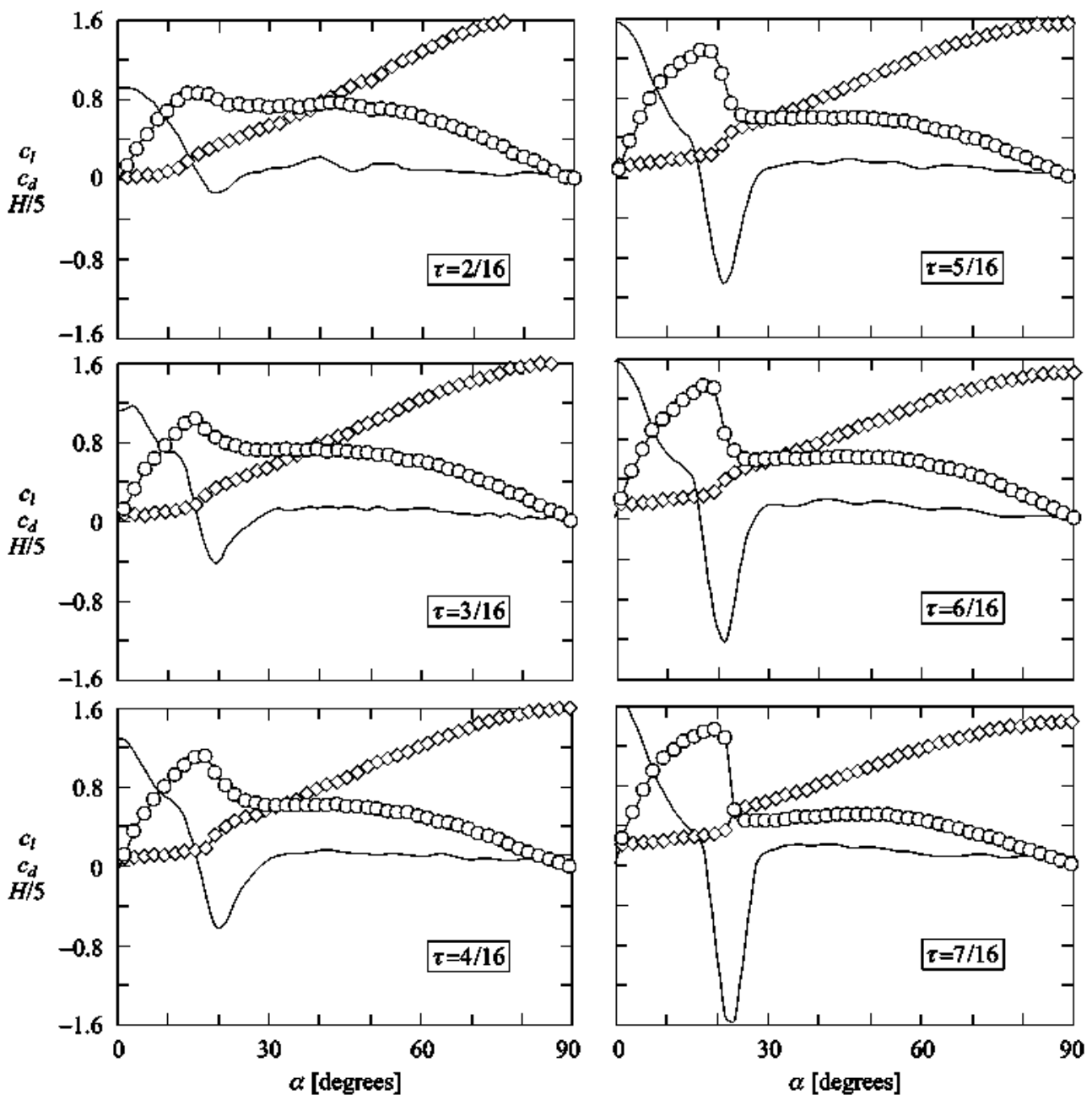

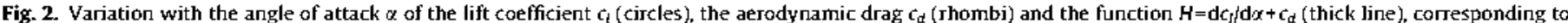
elliptical cross-section bodies at Reynolds number $R e \cong 0.75 \times 10^{5}$. The value of the relative thickness $t$ is indicated in the inserts. 


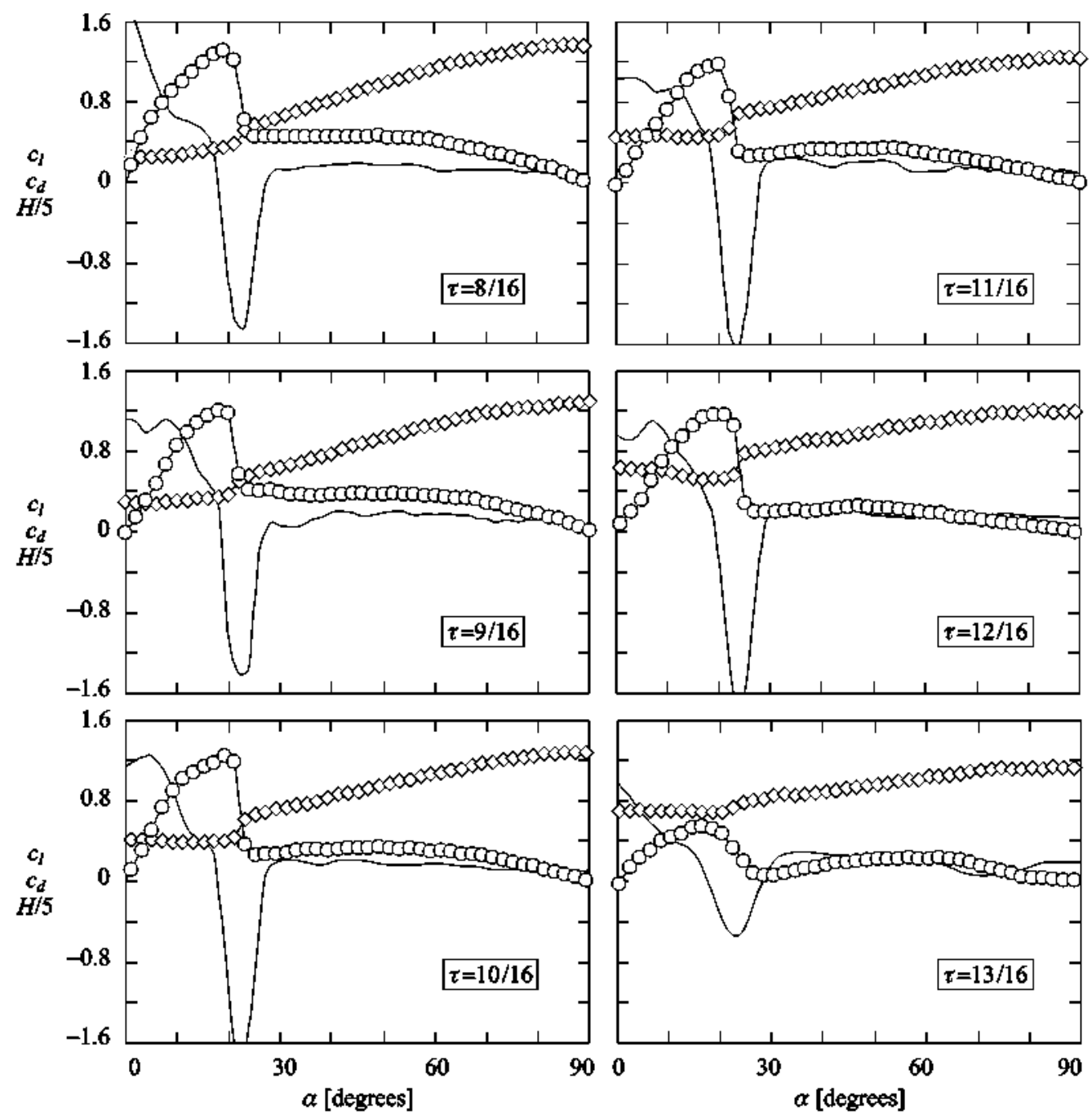

Fig. 2. (Continued)

cross-section, where boundary layer detachment, thus stalling, depends both on the pressure field on the body surface and on the flow regime, either laminar or turbulent, in the boundary layer. Amongst rounded bodies, one of the most suitable for systematic studies is the elliptical ones. Taking the relative thickness as the relevant geometrical parameter, it is clear that the galloping behaviour must be very different depending on its value. For a very small relative thickness, the galloping behaviour should resemble that of a flat plate, whereas for values close to unity the elliptical body should behave like a circular cross-section body. Besides its own interest as a fluid-structure interaction problem, elliptical cross-section bodies appear in a wide variety of engineering applications. These elliptical bodies can be found, for example, in reversible fans and in ship masts, but the main interest on the galloping behaviour of elliptic shapes is because of their use as sun-shadowing devices in some emblematic buildings. Large adjustable vertical elliptical louvers have been used in several buildings in Spain, and canopies made out of horizontal elliptical louvers extending the eaves of the roof of the Terminal-2 building of Heathrow Airport (London) are foreseen.

In this paper the galloping stability to cross-flow translational vibration of elliptical two-dimensional cross-section bodies has been analysed through wind tunnel experiments. Most of the experimental effort has been devoted to determine the variation with the angle of attack of both the lift coefficient and the aerodynamic drag coefficient curves. Twelve elliptical crosssection cylinders were tested, the relative thickness being $\tau=n / 16$, with $n=2,3, \ldots, 13$ (Fig. 1). Several testing conditions were considered, changing the upstream flow velocity (thus the Reynolds number) as well as the upstream turbulence intensity. Besides, to get additional insight, pressure distributions on a representative elliptical cylinder having $\tau=0.5$ were measured.

\section{Experimental set-up}

To experimentally determine the galloping features of elliptical cylinders, two different wind tunnels were used: one for net forces measurements at low Reynolds numbers and a second for pressure distribution measurements at higher Reynolds numbers and different turbulence intensities of the incoming flow.

Lift and drag aerodynamic coefficients were measured in the S1C wind tunnel of IDR/UPM. This wind tunnel is an open circuit two-dimensional wind tunnel with a test chamber $0.15 \mathrm{~m}$ width. $0.90 \mathrm{~m}$ high and $1.20 \mathrm{~m}$ long. Wind velocity profile at the model test section is uniform within $\pm 1 \%$, the turbulence intensity 
being around $3 \%$. The maximum wind velocity of the stream at the test section of the wind tunnel is $25 \mathrm{~ms}^{-1}$, which provides a Reynolds number based on the model characteristic length, $c=0.08 \mathrm{~m}$, of $\operatorname{Re}=1.4 \times 10^{5}$.

Aerodynamic loads were measured with a six-component strain-gauge balance (AT], model Gamma SI-130-10). The balance is mounted on a fixed reference frame in such a way that one of the balance axes becomes aligned with the upstream direction. The balance holds a rotating platform to which the model is attached through an appropriate fixture. The rotating platform allows setting the model angle of attack with $\pm 1^{\text {" accuracy. The }}$ balance has a maximum measurement uncertainty of $1.25 \%$. No blockage corrections of the measured results have been considered, as even in the worst case the blockage ratio was smaller than $9 \%$.

Once a selected model is attached to the rotating platform, angles of attack are varied from $\alpha=0-90^{\prime}$ at $2^{\prime \prime}$ steps, and at each step the six outputs coming from the balance are stored in a PC, as well as the dynamic pressure inside the test chamber (which is measured with an Air Flow 048 Pitor tube connected to a Schaewitz Lucas P-3061-2WD pressure transducer)

As mentioned before, the influence of the Reynolds number on the galloping phenomenon can be explained by taking into account the character either laminar or turbulent of the boundary layer on the elliptical-body surface at the forward edge. To check such behaviour, additional measurements were performed at the $\mathrm{A} 4 \mathrm{C}$ wind tunnel of IDR/UPM. This wind tunnel is $0.20 \mathrm{~m}$ width, $1.80 \mathrm{~m}$ height and $2.00 \mathrm{~m}$ long test chamber, where the upstream turbulence intensity can be modified by changing the porosity of the wire meshes that are placed at the contraction entrance. Results were obtained by using an elliptical cross-section model having a relative thickness of 0.5 . The model is $0.28 \mathrm{~m}$ chord and $0.20 \mathrm{~m}$ span, and it is equipped with 68 pressure taps distributed on the middle section of the body, evenly spaced. Pressure taps are connected to a pressure scanner, model ZOC 33/64PXX2 from Scanivalve. The model is mounted on a rotating platform ESP100/ RV80PP from Newport. The dynamic pressure in the test chamber is measured by a Pitot tube Air Flow 048 connected to a Schaewitz Lucas pressure transducer. During the experiments the angle of attack was swept from $0^{\circ}$ to $90^{\circ}$ at $2^{\circ}$ steps. All the experimental sequences, settling the different values of the angle of attack and pressure signals measurement and archiving, was controlled by a PC. It must be pointed out that with this experimental arrangement the blockage of the tests chamber becomes somewhat large for high values of the angle of attack (the blockage ratio changes from 0.08 at $\alpha=0^{\circ}$ to 0.16 at $\alpha=90^{\circ}$ ). Therefore, blockage corrections of the results were made accordingly (Barlow et al., 1999).

\section{Experimental results}

Galloping stability analysis has been performed according to standard procedures already used in galloping studies (Alonso and Meseguer, 2006; Alonso et al., 2009). Concerning Den Hartog criterion, from the $\mathrm{S1C}$ wind tunnel results the lift and drag coefficients were calculated from the appropriate outputs, and from these the function $H(\alpha, \tau, \operatorname{Re})=\mathrm{d} c_{i} / \mathrm{d} \alpha+c_{d}$ was determined. Some results are shown in Fig. 2, where the variation with the angle of attack of the lift coefficient, the drag coefficient and the function $H(\alpha, \tau, \operatorname{Re})$ has been represented. The results shown in Fig. 2 correspond to a Reynolds number Re $=0.75 \times 10^{5}$. Concerning the $c_{1}(\alpha)$ curves shown in Fig. 2 it must be pointed out the sudden transition in the aerodynamic characteristics that takes places at $\alpha \approx 20^{\circ}$ for a wide range of values of the relative thickness $\tau$, where a sudden drop of the lift coefficient occurs, so that the lift coefficient slope becomes largely negative, $\mathrm{d} c_{i} / \mathrm{d} \alpha \ll 0$. Such a drop of the lift slope becomes steeper as the thickness ratio $\tau$ increases and finally disappears as the body cross-section approaches the circular shape $(\tau=1)$. ln Fig. 2 experimental results are presented only for the range $0^{\prime \prime} \leq \alpha \leq 90^{\circ}$. Obviously, because of the symmetries of an elliptical cross-section shape, the lift force curves are antisymmetric with respect to both $\alpha=0^{\circ}$ and $90^{\circ}$, whereas the drag force ones are symmetric with respect to these values, $\alpha=0^{\circ}$ and $90^{\circ}$.

The galloping behaviour of elliptical cylinders at low values of the Reynolds number $\left(\mathrm{Re}<10^{5}\right.$ ) is summarized in Fig. 3, where the iso- $H$ curves corresponding to given negative values of the function $H$ (obtained from Fig. 2) have been represented in the $\alpha-\tau$ plane. Note that for very small values of the relative thickness, $\tau \approx 0$, the elliptical bodies behave like flat plates, although the lift coefficients of elliptical bodies at higher angles of attack are smaller than the ones reported for flat plates (Wick, 1954; Blevins, 1992), probably because the rounded edges of elliptical cylinders are less critical than the sharp edges of flat plates from the point of view of the boundary layer separation. From medium to high relative thickness of the elliptical cylinders, the value of the lift coefficient grows almost linearly with the angle of attack provided it is small enough, say $\alpha<5^{\circ}$ (Fig. 2), the lift coefficient curve slope being even slightly larger than $2 \pi$. As $\alpha$ grows the value of the lift curve slope decreases, but it is still positive. Due to adverse pressure gradients, close to $\alpha=20^{\circ}$ the laminar boundary layer separates near the leading edge. However, because of the transition to turbulent of the separated laminar flow, the shear layer reattaches on the body upper side and a recirculation bubble is formed close to the leading edge (Carmichael, 1981; O'Meara and Mueller, 1987; Schewe, 2001). To some extent such behaviour is similar to the one observed in medium and small thickness airfoils with a well defined trailing edge (McGullough and Gault, 1951; Torenbeek, 1982). The laminar recirculation bubble where boundary layer is separated clearly appears as a small but intense suction peak in the pressure distribution on the body surface (such a pressure peak is identified with the label "rb" in Fig. 4, where the point A indicates the reattachment point of the laminar recirculation

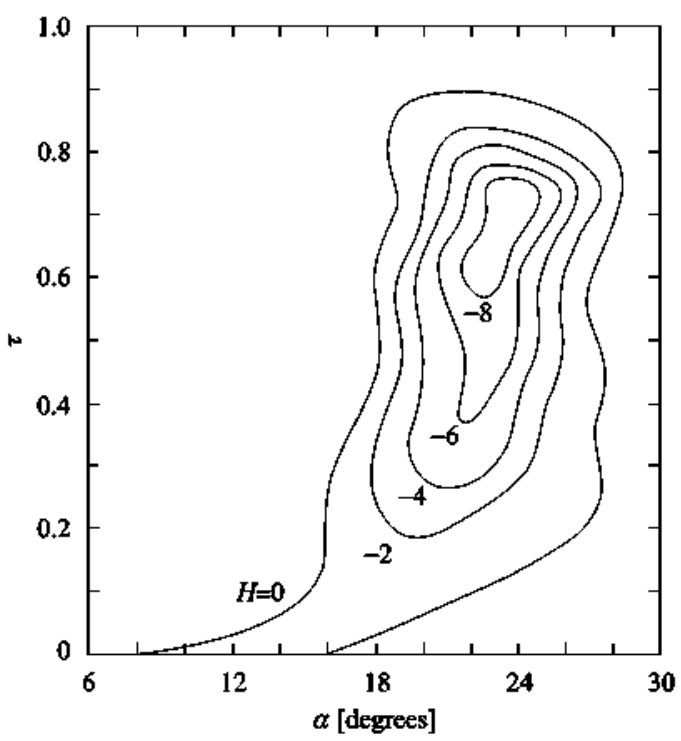

Fig. 3. Stability diagram in the angle of attack versus relative thickness plane $(\alpha, \tau)$ of elliptical cross-section cylinders. Results are based on static test results. Figures on the curves indicate the value of the function $H=d c_{f} d d \alpha+c_{d}$. 

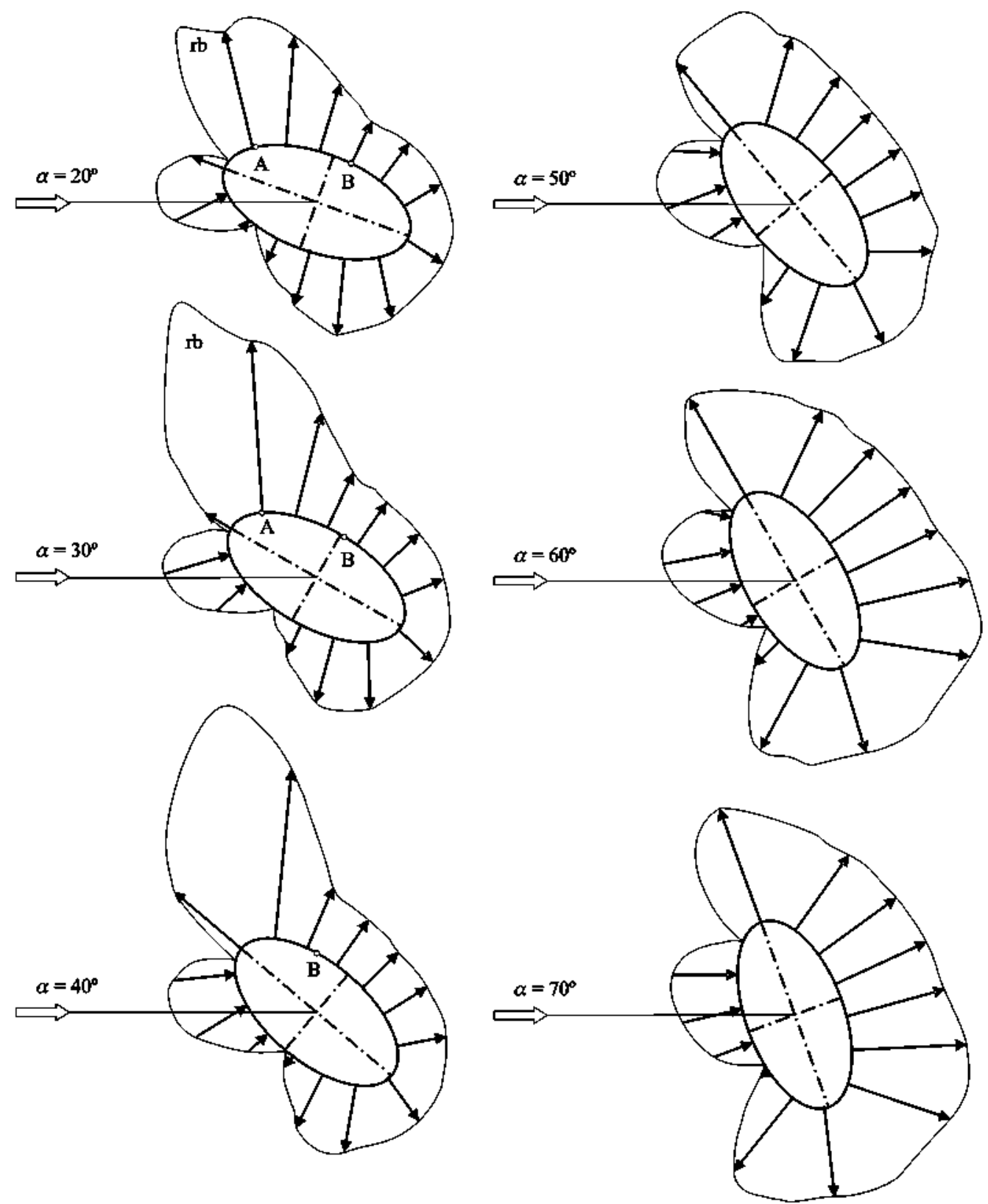

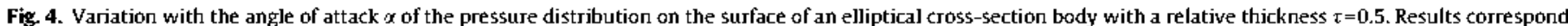

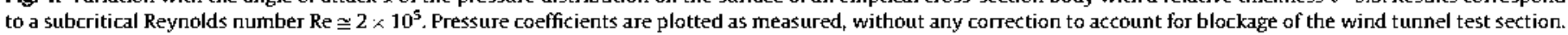

bubble. Of course the reattached (turbulent) boundary layer becomes again separated (point $B$ ) before reaching the trailing edge of the body. As the angle of attack grows the turbulent, second separation point $B$ moves towards the leading edge, but even in this range of values of the angle of incidence the lift stil] increases as $\alpha$ grows, as it can be deduced from the pressure distribution shown in Fig. 4. This behaviour ends when the turbulent separation point $B$ is close enough to the reattachment point of the laminar recirculation bubble, $A$, where both points $A$ and $B$ merge in a single point; the whole upper side boundary layer becomes separated, with a sudden decrease of the lift coefficient. Obviously, such a change of the lift coefficient is accompanied of a sudden increase of the drag coefficient.

The sudden fall of the lift coefficient value is more and more steeped as $\tau$ increases, although for large values of the relative thickness ( $\tau>0.8 \cong 13 / 16$ ), as the elliptical cross-section approaches to the circular one, the jump in the $c_{i}(\alpha)$ curves becomes smoother, and finally disappears (Fig. 3). The most unstable elliptical cylinders are those with a relative thickness close to 0.7 . A similar behaviour has been observed in biconvex airfoils with well defined wedged trailing and leading edges (Alonso et al., 2009), although in biconvex airfoils separation is due to the very sharp leading edge, which means an extremely large value of the adverse pressure gradient at the leading edge independently of the value of the angle of attack.

Obviously, since boundary layer features depend on the Reynolds number, it can be expected that the above described behaviour, driven by the existence of a recirculation bubble at the leading edge, be modified as the Reynolds number increases. Actually, for Reynolds numbers high enough the boundary layer transition from laminar to turbulent flow will take place before separation occurs, then the laminar separation will disappear and 

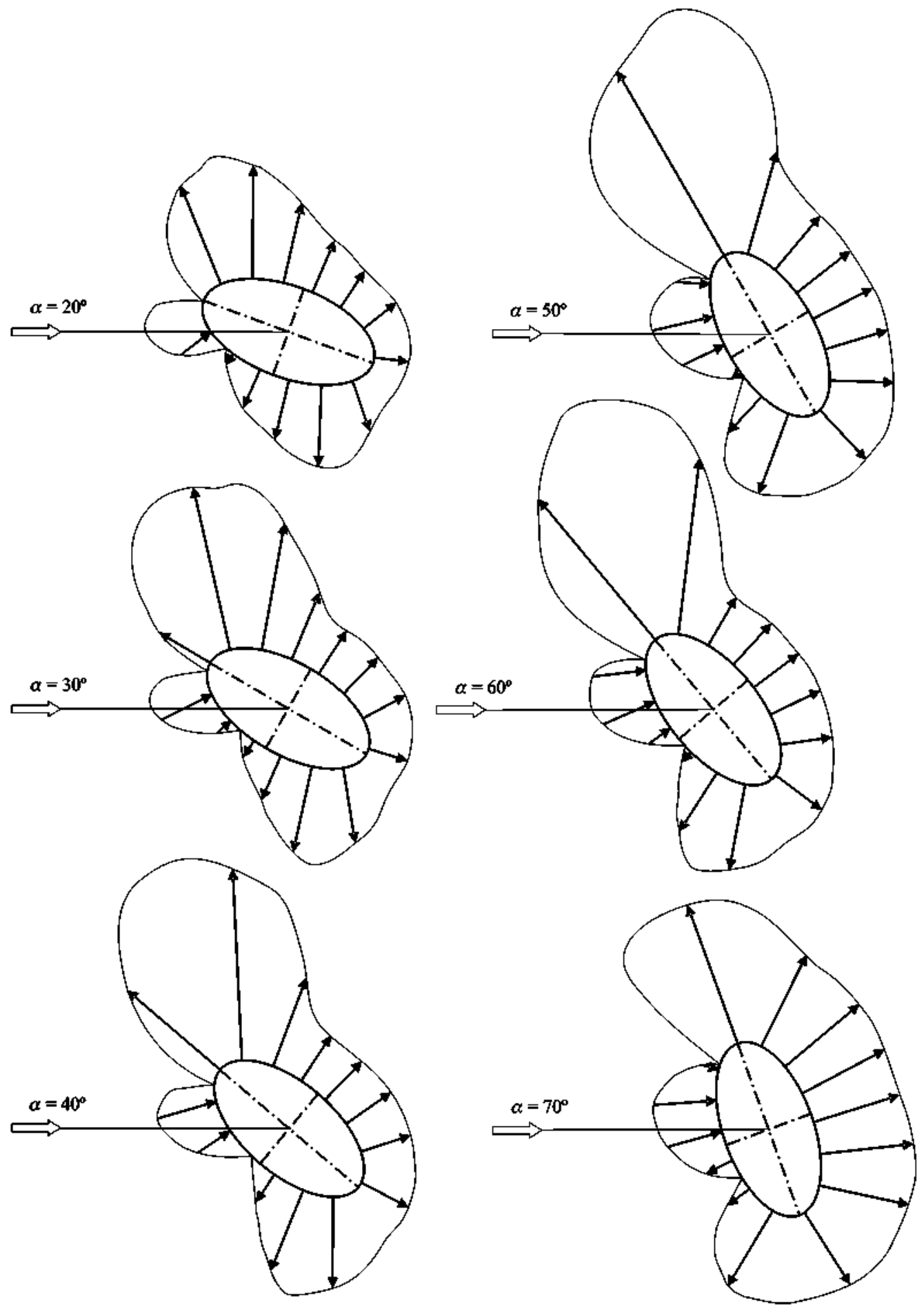

Fig. 5. Variation with the angle of attack $\alpha$ of the pressure distribution on the surface of an elliptical cross-section body with a relative thickness $\tau=0.5$. Results correspond to a supercritical Reynolds number $\operatorname{Re} \cong \mathbf{4} \times 10^{5}$. Pressure coefficients are plotted as measured, without any correction to account for blockage of the wind tunnel test section.

the galloping characteristics of elliptic cylinders will be modified. Aiming at checking such behaviour, additional tests were performed at different Reynolds numbers. The modification of this parameter was done by changing the wind speed (using the same $\mathrm{S} 1 \mathrm{C}$ wind tunnel) or by changing the model size (in this other case the $\mathrm{A} 4 \mathrm{C}$ wind tunnel was used). The pressure distributions on the body surface in the turbulent flow case (supercritical Reynolds number), for the same $\tau=0.5$ elliptical body are shown in Fig. 5, where results are represented for the same values of the angle of attack as in Fig. 4. Observe that now the absence of a laminar recirculation bubble strongly modifies the boundary layer separation behaviour. Since a turbulent boundary layer can withstand more severe adverse pressure gradients than a laminar one, the low pressure region close to the body leading edge is maintained even at large values of the angle of attack $\left(\alpha \cong 65^{\circ}\right)$ 

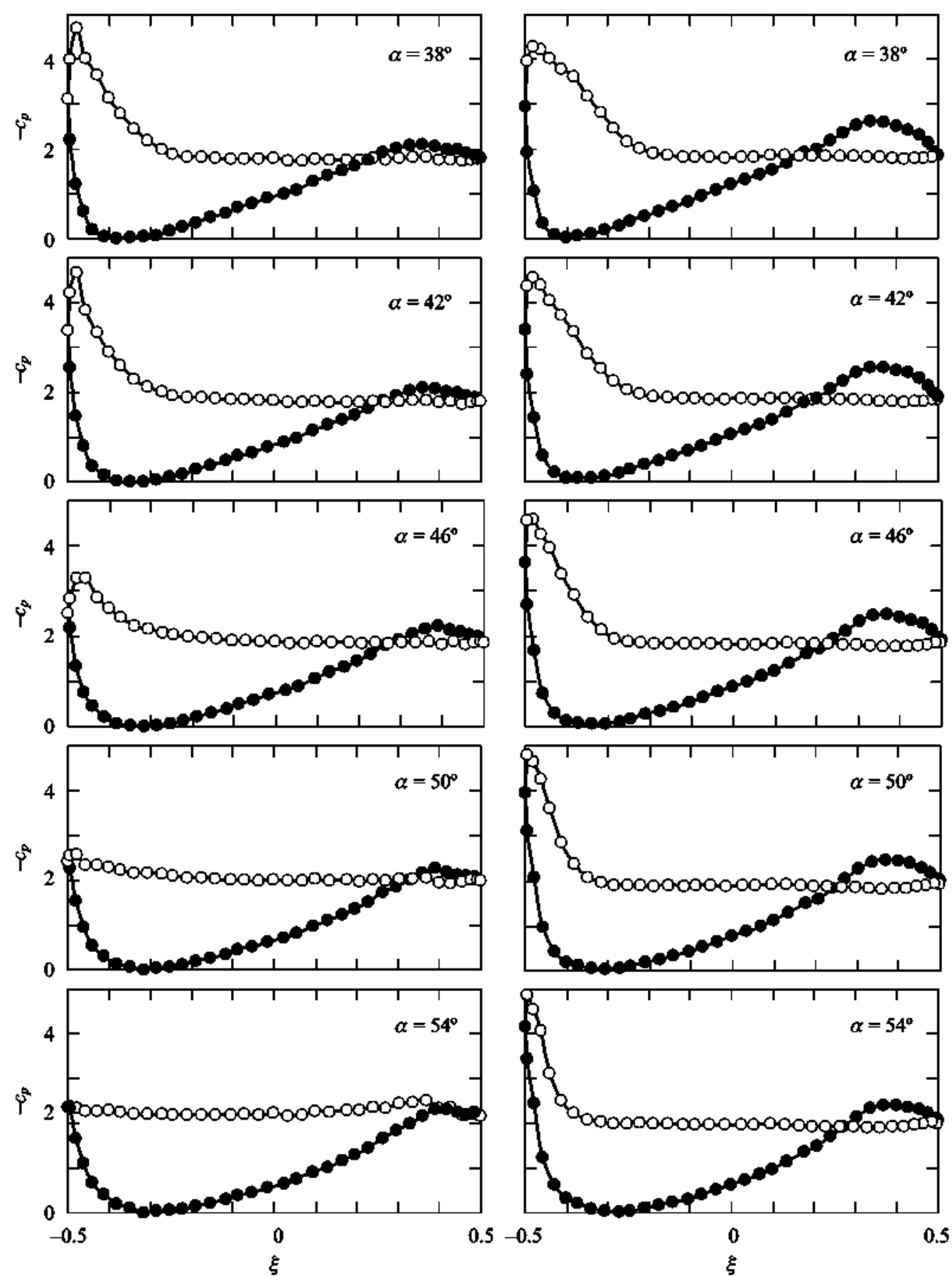

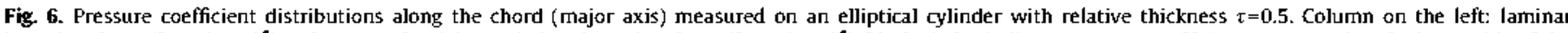

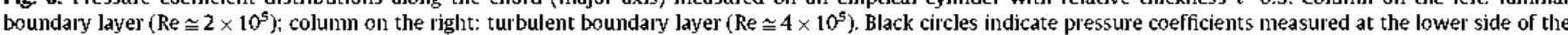
elliptical body. whereas white ones indicate pressure coefficients measured at the upper side.

To summarize the influence of the boundary layer regime on the aerodynamic forces acting on the body, pressure distributions along the body chord of the $\tau=0.5$ elliptical cylinder at two different Reynolds numbers and several angles of attack are also shown in Fig. 6 . The results correspond to the same test conditions to the ones shown in Figs. 4 and 5, but now considering a narrow range of angles of attack around the value of the angle of attack at which the elliptical cross-section body stalls in the laminar boundary layer case. Results on the left column correspond to an incident flow at Reynolds number $\operatorname{Re} \bumpeq 2 \times 10^{5}$, and turbulence intensity $<1 \%$, whereas those on the right column are for $\operatorname{Re} \cong 4 \times 10^{5}$ and a turbulence intensity of some 3\%. It must be stressed that in all cases the distance from the forward stagnation point (at the body lower side) to the trailing edge of the elliptical cylinder is much larger than the distance from the forward stagnation point to the leading edge. Therefore, in the lower side boundary layer transition occurs before the boundary layer separation, whereas at the upper side the boundary layer approaching the separation point can be laminar or turbulent depending on the values of both the Reynolds number and the free-flow turbulence intensity.

By comparing both columns in Fig. 6 one can realize again the dependence of the stalling process on the character either laminar or turbulent of the boundary layer at the leading edge. 

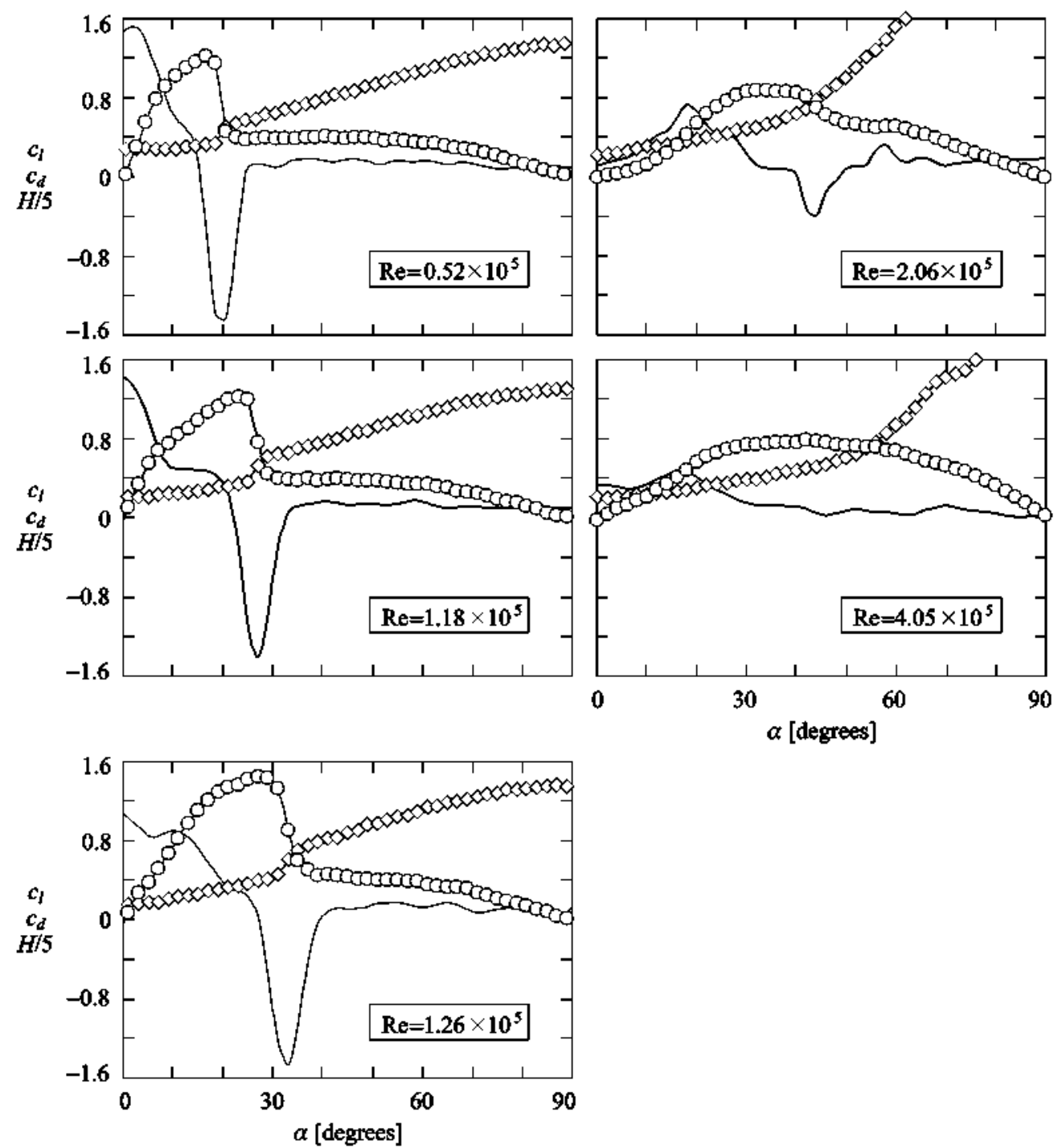

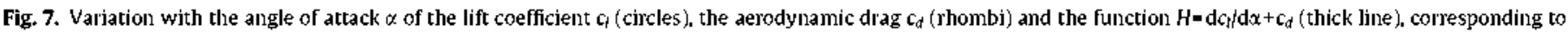
elliptical cross-section bodies with relative thickness $\tau=0.5$ at different Reynolds number, as indicated in the inserts.

At low Reynolds number a laminar separation bubble is formed at the leading edge, which can be identified by the narrow and intense suction peak $\left(-c_{p}>3.0\right)$ appearing in the $\alpha=38^{\circ}$ and $42^{\circ}$ pressure distributions of the first column in Fig. 6 . As already stated, downstream of such a suction peak the boundary layer, now turbulent, is reattached, and it separates again provided the angle of attack is high enough. Such second separation point moves to the leading edge as $\alpha$ grows, until it reaches the laminar bubble reattachment point. When this happens the leading edge suction peak suddenly disappears $\left(\alpha \approx 45^{\circ}\right)$, and beyond this threshold value of the angle of attack the whole upper side of the $\tau=0.5$ elliptical cylinder becomes stalled. As a consequence of the loss of the leading edge suction peak, the lift coefficient curve decreases by a valuable amount in a narrow gap of angles of attack.

In the turbulent boundary layer case the boundary layer can withstand the adverse pressure gradients appearing at the leading edge without boundary layer separation. The forward-edge suction peak remains at higher values of the angle of attack than in the laminar case and the decreasing in the lift coefficient curve becomes smoother. Note that the suction peak of the leading edge becomes narrower, but at the same time its intensity increases (the turbulent suction peak does not disappears until $\alpha \cong 65^{\circ}$ ). Therefore, although the lift coefficient curve decreases, the slope $\mathrm{dc}_{i} / \mathrm{d} \alpha$ is less negative in the turbulent boundary layer case than in the laminar one. Besides, since boundary layer separation is delayed, the drag coefficient is also smaller (a non-fully separated boundary layer means a narrow wake behind the body), but usually the decrease of the body drag is less pronounced than the decrease of the lift slope, and then the function $H=\mathrm{d} c_{l} / \mathrm{d} \alpha+c_{d}$ becomes larger, and it can be even positive in the whole range of the angles of attack as the Reynolds number grows. This implies that galloping instability can disappear provided the Reynolds number is high enough.

To illustrate the above described behaviour, the aerodynamic forces measured at different Reynolds numbers are depicted in Fig. 7, where the dependence on the angle of attack of the lift coefficient, the aerodynamic drag coefficient, and the stability function $H$ are shown for different values of the Reynolds number. Such results were obtained for an elliptical cylinder with a relative thickness $\tau=0.5$. As the Reynolds number grows the lift jump appears at higher values of the angle of attack, and finally disappears, provided Re is high enough. Unfortunately, there is a gap in the Reynolds numbers of the experimental results 


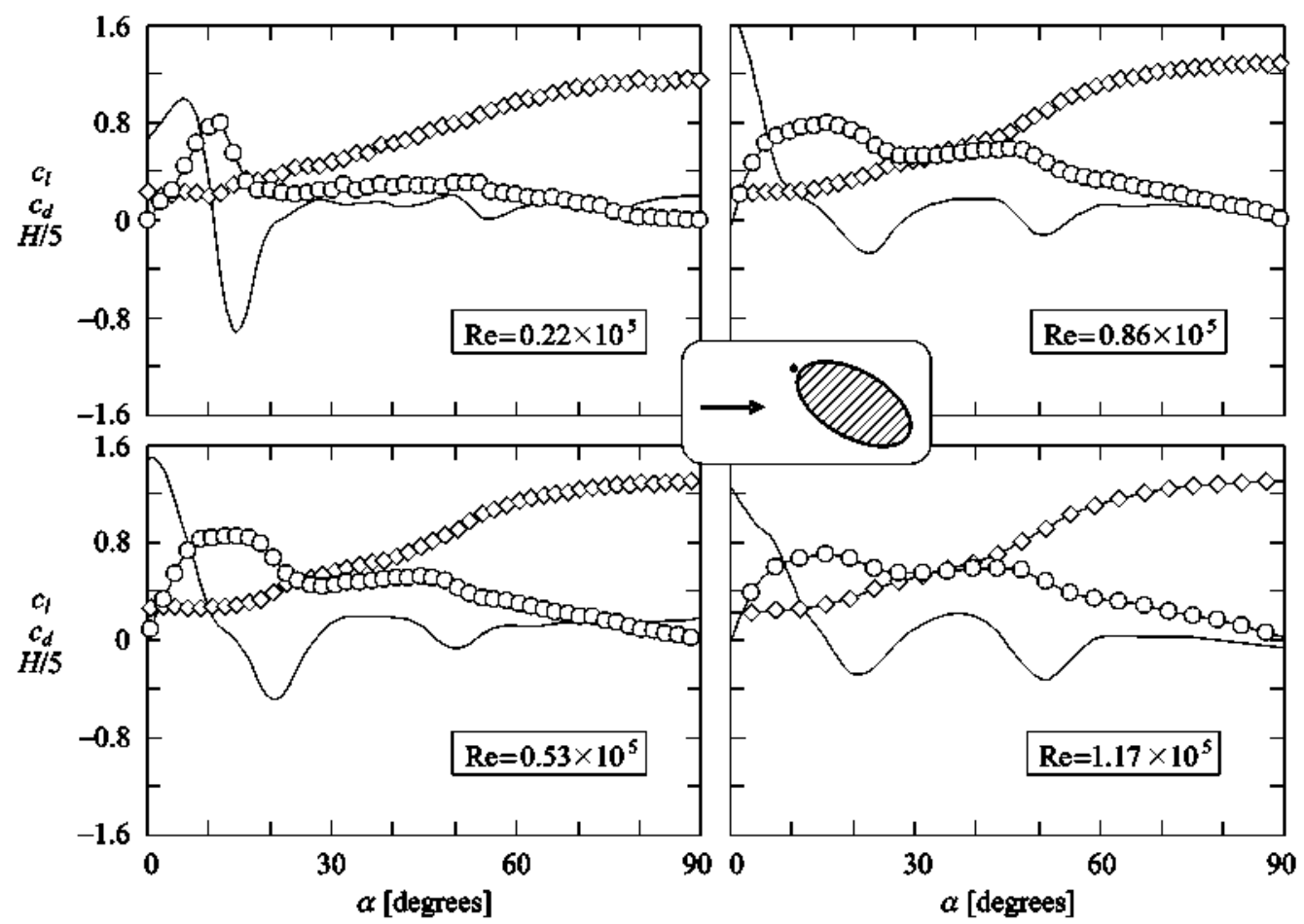

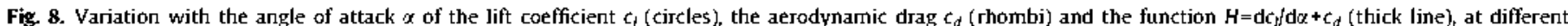

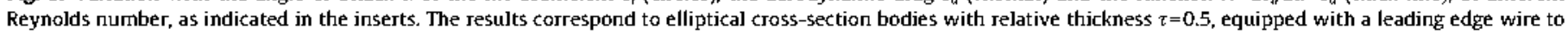
force boundary layer transition.

measured at each one of the wind tunnels, because the performances of the wind tunnels do not overlap. However, it is clear that if the Reynolds number is high enough and the boundary layer becomes turbulent, elliptical bodies with $\tau=0.5$ do not show transverse galloping. In the turbulent boundary layer case the separation starts at the trailing edge, the separation point moving towards the leading edge as the angle of attack grows (Fig. 5), which is the typical stall behaviour of thick airfoils.

The same conclusion can be derived by forcing the boundary layer transition at low Reynolds numbers by adding some turbulence generator at the leading edge. In a new set of tests performed in the S1C wind tunnel, the $\tau=0.5$ elliptical cylinder was equipped with a wire $1 \mathrm{~mm}$ in diameter placed $2 \mathrm{~mm}$ in front of and parallel to the body leading edge. The wire generates a vortex wake that trigger the transition of the boundary layer on the elliptical cylinder, even at low values of the Reynolds number (Meseguer et al., 2008). The results obtained with this new configuration are shown in Fig. 8. Note that lift curves are now smoother than in the clean cylinder case. There is still an instability zone for angles of attack close to $\alpha=20^{\circ}$, but the instability is now less severe than the one appearing without leading edge wire. Besides, an almost marginal new instability zone appears at large angles of attack $\left(\alpha \approx 50^{\circ}\right)$.

Form the results shown in Figs. 7 and 8 the stability maps in the $\alpha$-Re plane displayed in Fig. 9 were obtained. The upper plot corresponds to the clean elliptical cylinder whereas the lower one corresponds to the body equipped with a leading edge wire.

\section{Conclusions}

Through wind tunnel testing the galloping instability characteristics of elliptical cross-section bodies have been analysed for angles of attack ranging from $0^{\circ}$ to $90^{\circ}$. Galloping instability zones have been identified and represented in stability maps, using as parameters the angle of attack, the body's relative thickness and the Reynolds number.

Plotting the instability regions in the $\alpha-\tau$ plane, at low values of the Reynolds number ( $R e<10^{5}$ ), it can be observed how the behaviour of the elliptical cylinders evolves from very small values of the relative thickness, $\tau \approx 0$ (a flat plate like behaviour) up to large values of that relative thickness $(\tau>0.8 \cong 13 / 16$ ), as the elliptical cross-section approaches to the circular one, reaching therefore galloping stability. Intermediate values of $\tau$ show similar instability patterns, being remarkable the sudden drop of the lift coefficient that takes places at $\alpha \approx 20^{\circ}$ for those values of the relative thickness $\tau$, causing the galloping instability. Largest instability is found for elliptical cylinders with a relative thickness close to 0.7 ( $\tau \cong 11 / 16$ ). This phenomenon is explained by the flow morphology around the body, in particular by the existence of a recirculation bubble at the leading edge.

The dependency of galloping instability with Reynolds number has also been shown: the boundary layer transition from laminar to turbulent flow is modified as the Reynolds number increases, and at a certain value of Reynolds this transition takes place before separation occurs, changing therefore the galloping characteristics of these elliptical cylinders. In particular, experimental results here reported seem to indicate that elliptical bodies with $\tau=0.5$ are stable from the point of view of transverse galloping at any angle of attack for Reynolds numbers above a certain value when the boundary layer becomes turbulent. Measured pressure distributions along the surface of the $\tau=0.5$ elliptical cylinder at several angles of attack and two different Reynolds numbers support this conclusion.

At low Reynolds numbers, if the boundary layer transition is enforced by adding for instance some turbulence generator at the leading edge, the same behaviour is found for the elliptical cylinders (Figs. 8 and 9). This type of vortex generation device could be used therefore in situations where the geometry of the 

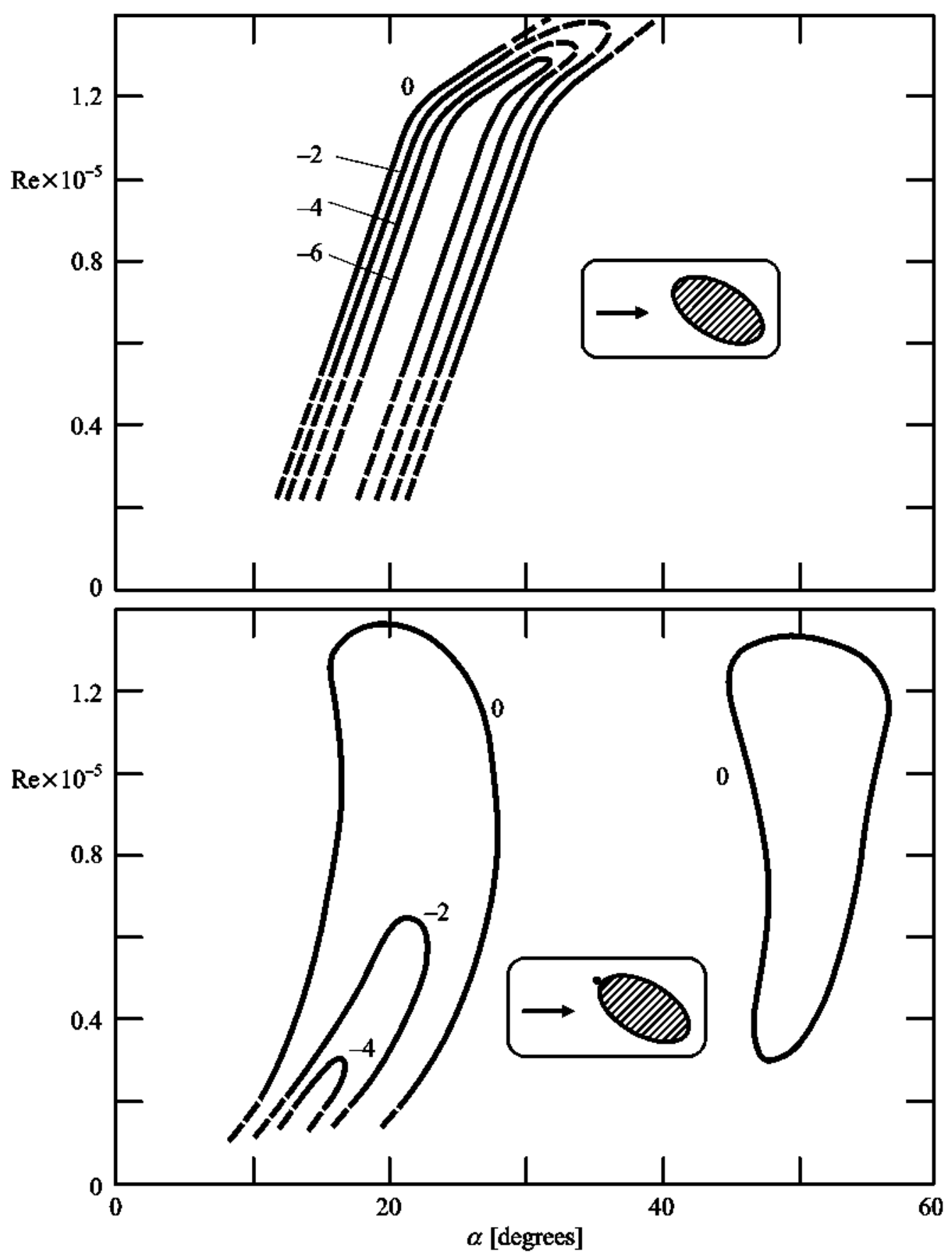

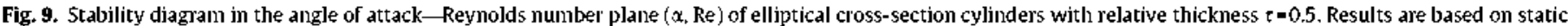

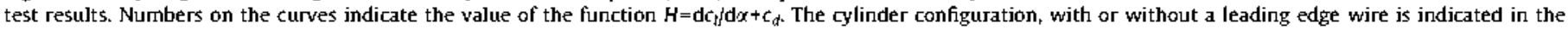
inserts.

body's cross-section and the incident wind velocity and angle of incidence cause galloping stability problems.

\section{References}

Alonso, G., Meseguer, J., 2006. A parametric study of the galloping instability of triangular cross-section bodies. J. Wind Eng. Ind. Aerodyn. 94, 241-253.

Alonso, G. Meseguer, J. Pérez-Grande, l., 2005. Galloping 0scillations of twodimensional triangular cross-sectional bodies. Exp. Fluids 38, 789-795.

Alonso, G. Meseguer, J. Pérez-Grande, I., 2007, Galloping stability of triangular cross-sectional bodies: a systematic approach. J. Wind Eng. Ind. Aerodyn. 95 , 928-940.
Alonso, G. Valero, E., Meseguer. J., 2009. An analysis on the dependence on cross section geometry of galloping stability of two-dimensional bodies having either biconvex or rhomboidal cross sections. Eur. J. Mech. B Fluids $28,328-334$.

Barlow, J.B., Rae, W.H., Pope, A., 1999. Low-Speed Wind Tunnel Testing. Wiley, New York.

Blevins, R.D., 1992. Applied Fluid Dynamics Handbook. Krieger, Malabar.

Blevins, R.D., 2001. Flow-lnduced Vibrations, 2nd ed Krieger, Malabar.

Carmichael, B.H., 1981. Low Reynolds number airfoil survey, vol, 1, NASA CR 165803.

Den Hartog. J.P., 1956. Mechanical Vibrations, 4th ed McGraw-Hill, New York. Hémon, P., Santi, F., 2002. On the aeroelastic behaviour of rectangular cylinders in cross-flow. J. Fluids 5truct. 16 (7), 855-889.

McGullough, G.B., Gault, D.E., 1951. Examples of three representative types of airfoil-section stall at low speed. NACA TN 2502. 
Meseguer, J., Sanz-Andrés, A., Pérez-Grande, I., Franchini, S., Sanz, J.L., Ortega, F., Chiappe, L, 2008. On the use of tubulence generators as high-lift devices in the flight at low Reynolds numbers. Proc. MMechE Part G J. Aerosp. Eng. 222, 1007-1013.

Naudascher, E., Rockwell, D., 1994. Flow-Induced Vibrations. Dover, New York.

o'Meara, M.M., Mueller, T.J., 1987. laminar separation bubble characteristics on an airfoil at low Reynolds numbers. AlAA J. 25, 1033-1041.
Schewe, G., 2001. Reynolds-number effects in flow around more-or-less bluff bodies. J. Wind Eng. Ind. Aerodyn. 89, 1267-1289.

Torenbeek, E., 1982. Synthesis of Subsonic Airplane Design. Delft University Press, Kluwer Academic Publishers, Dordrecht.

Wick, B.H. 1954. Study of the subsonic forces and moments on an inclined plate of infinite span. NACA TN 3221 